\title{
Erratum to: PIGN mutations cause congenital anomalies, developmental delay, hypotonia, epilepsy, and progressive cerebellar atrophy
}

\author{
Chihiro Ohba • Nobuhiko Okamoto • Yoshiko Murakami • Yasuhiro Suzuki • \\ Yoshinori Tsurusaki • Mitsuko Nakashima • Noriko Miyake • Fumiaki Tanaka • \\ Taroh Kinoshita • Naomichi Matsumoto $\cdot$ Hirotomo Saitsu
}

Received: 9 March 2014 / Accepted: 11 March 2014 / Published online: 19 March 2014

(C) Springer-Verlag Berlin Heidelberg 2014

\section{Erratum to: Neurogenetics}

DOI 10.1007/s10048-013-0384-7

In abstract and Figure 1 of our paper " $P I G N$ mutations cause congenital anomalies, developmental delay, hypotonia, epilepsy, and progressive cerebellar atrophy (DOI: 10.1007/s10048-0130384-7)" we erroneously described the mutation as c.808C $>\mathrm{T}$, instead of c.808T $>$ C. We apologize for this mistake.

The online version of the original article can be found at http://dx.doi.org/ 10.1007/s10048-013-0384-7.

C. Ohba $\cdot$ Y. Tsurusaki $\cdot$ M. Nakashima $\cdot$ N. Miyake $\cdot$

N. Matsumoto $\cdot$ H. Saitsu $(\square)$

Department of Human Genetics, Graduate School of Medicine,

Yokohama City University, Yokohama 236-0004, Japan

e-mail: hsaitsu@yokohama-cu.ac.jp

C. Ohba $\cdot$ F. Tanaka

Department of Clinical Neurology and Stroke Medicine, Yokohama

City University, Yokohama 236-0004, Japan

N. Okamoto

Department of Medical Genetics, Osaka Medical Center and

Research Institute for Maternal and Child Health, Izumi 594-1101,

Japan

Y. Murakami · T. Kinoshita

Department of Immunoregulation, Research Institute for Microbial

Diseases, Osaka University, Osaka 565-0871, Japan

Y. Murakami • T. Kinoshita

World Premier International Immunology Frontier Research Center,

Osaka University, Osaka 565-0871, Japan

\section{Y. Suzuki}

Department of Pediatric Neurology, Osaka Medical Center and

Research Institute for Maternal and Child Health, Osaka, Japan 